\title{
Production and quality evaluation of low glycemic index crispy waffle from whole wheat flour supplemented with type 4-resistant starch and sacred lotus stamen
}

\author{
${ }^{1}$ Kaewmak, N., ${ }^{1}$ Chupeerach, C., ${ }^{1}$ Suttisansanee, U., ${ }^{2}$ Siriwan, D., ${ }^{1}$ Chamchan, R., \\ ${ }^{1}$ Khemthong, C. and ${ }^{1}$ On-nom, N. \\ ${ }^{1}$ Institute of Nutrition, Mahidol University, 999 Phutthamonthon 4Rd., Nakhon Pathom, 7317, Thailand \\ ${ }^{2}$ Institute of Food Research and Product Development, Kasetsart University, Bangkok, 10900, Thailand
}

\author{
Article history: \\ Received: 23 May 2020 \\ Received in revised form: 30 \\ June 2020 \\ Accepted: 12 September 2020 \\ Available Online: 6 \\ December 2020
}

\section{Keywords:}

Type IV resistant starch (RS IV),

Sacred lotus stamen, Antioxidant activities, Total phenolic contents, Glycemic index (GI)

DOI:

https://doi.org/10.26656/fr.2017.4(S4).007

\begin{abstract}
Health and wellness are a trend observed throughout all types of food. Thus, this study aimed to develop a low glycemic index (GI) crispy waffle from whole wheat flour (WF) supplemented with type 4-resistant starch (RS IV) and sacred lotus stamen (SLSP). The low GI crispy waffle was formulated by substituting WF flour with RS IV at 35, 45 and $55 \%(\mathrm{w} / \mathrm{w})$. Then, the formula with the highest overall liking score was continuing to develop by replacing WF with SLSP at 30, 35 and $40 \%(\mathrm{w} / \mathrm{w})$. The physical and sensory qualities were determined. Moreover, the low GI crispy waffle with SLSP was evaluated on nutritional values, GI, total phenolic contents (TPCs) and antioxidant activities. The results showed that low GI crispy waffle with SLSP could successfully be developed by substituted WF with 55 and $18 \%$ (w/w) of RS IV and SLSP, respectively. The nutritional value of the developed product in $100 \mathrm{~g}$ was $491.22 \mathrm{kcal}, 64.52 \mathrm{~g}$ carbohydrate, $6.63 \mathrm{~g}$ protein, $22.96 \mathrm{~g}$ fat and $28.04 \mathrm{~g}$ fiber. Therefore, this product could be claimed as high fiber and classified as low GI product (28.8). In addition, the TPCs and antioxidant activities (by DPPH, FRAP and ORAC assays) of low GI crispy waffle with SLSP was $2.30 \mathrm{mg} \mathrm{GAE} / \mathrm{g}$ DW, $0.76 \mu \mathrm{mol} \mathrm{TE} / 100 \mathrm{~g}$ DW, $7.33 \mu \mathrm{mol} \mathrm{TE} / \mathrm{g}$ DW and $56.45 \mu \mathrm{mol} \mathrm{TE} /$ g DW, respectively. Therefore, it seemed that RS IV and SLSP could enhance the nutritional quality of the product with the consumer acceptability.
\end{abstract}

\section{Introduction}

The reformulation products for optimal nutritional value and making them as tasty as or better than the original is being a challenge to the food industry. One way to achieve a healthy product is to reduce of the calorie-laden ingredients, such carbohydrate and increased fiber. Carbohydrate-rich foods such as whole wheat flour (WF) have a high glycemic index (GI) and are not recommended for people with diabetes (BrandMiller et al., 2007; Choi et al., 2009). The concept of GI is the classification of carbohydrate-based foods according to their postprandial glucose responses. GI can be classified into 3 groups are high GI $(\geq 70)$, moderate GI (56-69) and low GI $(\leq 55)(3)$. Therefore, nutritional advice for people with diabetes promotes the consumption of low GI food in several countries such as the United Kingdom, and Australia (Perlstein, Hines and Milsavljevic, 1997; Nutrition Subcommittee of the Diabetes Care Advisory Committee of Diabetes UK, 2013).
Resistant starches (RS) are starches that are not able to be digested by enzymes in the small intestine and are fermented in the colon by microbiota (Birt et al., 2013). While RS can be found naturally in food products like less-ripe bananas, legumes and potatoes (RS I and II) (Lockyer and Nugent, 2017), there are many different types of RS developed through starch modification for use in food production (RS III and IV). RS are a valuable ingredient solution for product developers looking to enhance the fiber content of food products (Office of Nutrition and Food Labeling, Center for Food Safety and Applied Nutrition, Food and Drug Administration, US Department of Health and Human Services, 2016). RS have a mild flavor, are naturally white in color, and can be made either soluble or insoluble in water (Homayouni et al., 2014). When added to food products in place of traditional fibers, RS can produce a better appearance, texture and mouthfeel (Charalampopoulos et al., 2002). Baixauli et al. (2008) substituted up to $20 \%$ RS for traditional starch in a baked application with no significant difference in taste or acceptability. Moreover, 
Stewart et al. (2018) found that the replacement WF with RS IV (chemically modified starch) in scone can improve postprandial glucose and insulin sensitivity. Considered as a high non-starch polysaccharide resource, it can be developed for a functional food especially for its low GI.

Sacred lotus (Nelumbo nucifera) is an aquatic plant in the family Nelumbonaceae. The edible parts of lotus include the flowers, seeds, leaves, buds and rhizomes have been used in traditional Asian medicine for diverse disorders such as hypertension, vomiting, depression, skin diseases, heart problems, diarrhea and insomnia (Fan, 2017). In addition, the leaves, petal and stamen of lotus have investigated for biological activities against oxidative stress, cancer, diabetes and obesity. According to Jung et al. (2003), it found that the stamen contains a high bioactive compound (total phenolic compound) and antioxidant activity. These compounds are associated with health benefits such as a lower risk of developing cardiovascular disease, certain kinds of cancer and agerelated degenerative processes (Hung et al., 2004; Soerjomataram et al., 2010; Wang et al., 2011). Therefore, it could be used as a functional ingredient in product development.

Crispy waffle is one popular product which contains three main ingredients including WF, sugar and fat. Overconsumption may contribute to chronic diseases. Therefore, the objective of this study was to develop a low GI crispy waffle from WF supplemented with RS IV and SLSP. In the process, physical and sensory properties were evaluated. Then, the best formula was selected to test the nutritional values, GI, total phenolic contents (TPCs) and antioxidant activities.

\section{Material and methods}

\subsection{Raw materials}

The ingredients used in this study are WF (UFM Food Centre Co., Ltd., Bangkok, Thailand), sugar (Mitr Phol Sugar Corp., Ltd.), hen eggs (Charoen Pokphand Foods Public Co., Ltd.), butter and baking powder (KCG Corporation Co., Ltd.), cinnamon powder (Continental Food Co., Ltd.), yeast (Great hill Co., Ltd.), cow milk (Friesland Campina (Fresh) Thailand Co., Ltd.) and SLSP (Khwan-Phayao small and micro community enterprise, Phayao, Thailand). Moreover, RS IV which were modified from tapioca starch (the amount of fiber and starch of RS IV was $79.9 \mathrm{~g}$ and $7.9 \mathrm{~g}$ per $100 \mathrm{~g}$, respectively) were obtained from National starch and chemical Thailand Co., Ltd.

\subsection{Preparation of crispy waffle}

The formulas of crispy waffle were modified from the crispy waffle recipe by Chef Rudolph van Veen as shown in Table 1 . The preparation of crispy waffle was done by mixing all ingredients together. The mixture was kneaded properly until soft dough was obtained. $15 \mathrm{~g}$ of dough was cut equally and made into round shape. Before baking, the baking tong (FR-C11, FRY KING, Thailand) was preheated up to $140 \pm 5^{\circ} \mathrm{C}$. For baking, the dough was deposited on the bottom baking plate, the baking plate was closed, and the baking process started immediately (140 s). Then, the tong was opened the waffle was removed from the baking. Finally, the baked waffles were cut into round shape measuring $8 \mathrm{~cm}$. The three formulas were used for sensory evaluation. The formula which obtained the highest overall liking score was selected to be the control formula.

Table 1. Ingredients of three different crispy waffle formulas.

\begin{tabular}{lccc}
\hline \multirow{2}{*}{ Ingredients (\%) } & \multicolumn{3}{c}{ Formulas } \\
\cline { 2 - 4 } & $\mathrm{A}$ & $\mathrm{B}$ & $\mathrm{C}$ \\
\hline Wheat flour & 48.49 & 50.97 & 45.63 \\
Eggs & 5.82 & 7.84 & 16.73 \\
Butter & 24.27 & 19.59 & 21.3 \\
Brown sugar & 14.54 & 12.55 & - \\
Refined sugar & - & - & 15.96 \\
Cow milk & 5.82 & 7.85 & - \\
Yeast & 0.87 & - & - \\
Baking powder & - & 1.02 & - \\
Cinnamon powder & 0.19 & 0.19 & 0.38 \\
\hline
\end{tabular}

2.3 Preparation of low glycemic index (GI) crispy waffle from $R S I V$

The control crispy waffle formula (from previous experiment) was used to prepare low GI crispy waffle by partially replacing WF with RS IV at three different levels $(35,45$ and $55 \%(\mathrm{w} / \mathrm{w}))$. Other ingredients were the same. Then, the process of low GI crispy waffle preparation was same as the previous experiment. Physical and sensory properties of these samples were determined. The low GI crispy waffle formula which obtained the highest overall liking score was selected for the future study.

\subsection{Preparation of low GI crispy waffle fortified with sacred lotus stamen powder (SLSP)}

The low GI crispy waffle formula which was selected from the previous study was used to prepare low GI crispy waffle fortified with SLSP. Three different levels of SLSP which were 30,35 and $40 \%(w / w)$ were used to replace WF while other ingredients were the same. The preparation process was also the same as the previous study. Then, physical and sensory properties of these samples were determined. The low GI crispy waffle fortified with SLSP formula which obtained the highest overall liking score was continuing on nutritive values, GI, TPCs and antioxidant activities 
determination.

\subsection{Sensory analysis}

Sensory evaluation was conducted with thrity untrained panelists comprised of faculty members, staffs and graduate students of the Institute of Nutrition, Mahidol University (INMU), Thailand. The test was performed in an individual testing booth under the daylight-fluorescent lights of the sensory science laboratory at INMU. All samples were prepared one day before testing and kept in aluminum foil bags at $4 \pm 1{ }^{\circ} \mathrm{C}$. Samples were coded using random three-digit numbers. Panelists were provided with a glass of water and, instructed to rinse and swallow water between samples. They were given written instructions and asked to evaluate the products for acceptability based on its appearance, color, flavor, taste, texture and overall liking using nine-point hedonic scale $(1=$ dislike extremely to 9 = like extremely) (Meilgaard et al., 1999). The protocol was approved by Mahidol University Ethical Board (MU-CIRB) (No. MU-CIRB 2019/292.0811).

\subsection{Physical analysis}

The color values of sample were determined using Hunter Lab Digital Colorimeter (COLORFLEX 4510 model, USA). The CIE color values were recorded as $L^{*}$ (lightness), $a^{*}$ (redness) and $b^{*}$ (yellowness). For water activity $\left(\mathrm{A}_{\mathrm{w}}\right), \mathrm{A}_{\mathrm{w}}$ was determined using a portable water activity meter set $A_{w}$ (model ms 1, Novasina, Switzerland). Texture analysis of samples, hardness and fracturability were performed at ambient temperature (approximately $25 \pm 2^{\circ} \mathrm{C}$ ) using texture analyzer (TA.XT plus, Stable Micro Systems Ltd, YL, UK) equipped with a 3-Point Bending Rig (HDP/3PB) as according to the method described by Jambrec et al. (2013).

\subsection{Nutritive values and In vitro estimation of glycemic index (eGI) analysis}

The nutritive values of the control and the developed crispy waffle were determined according to AOAC (2016) including of energy, carbohydrate, protein, moisture content, total fat, ash and total fiber. The analytical procedures were performed by the Food and Nutrition Laboratory of the Institute of Nutrition, Mahidol University, Thailand.

In vitro estimation of glycemic index (eGI) in the control and the developed crispy waffle were determined according to Granfeldt et al. (1994) with slight modification. Using the hydrolysis curve (0-120 min), the hydrolysis index (HI) was calculated as the percentage of total glucose released from the samples, to that released from white bread. The glycemic index of the samples was estimated according to the equation:
$8.198+0.862 * \mathrm{HI}$.

\subsection{Total phenolic contents and antioxidant activities}

Approximately $0.5 \mathrm{~g}$ of sample was mixed with (10 $\mathrm{mL}) \quad 40 \%(\mathrm{v} / \mathrm{v})$ aqueous ethanol. After thoroughly mixing $(1 \mathrm{~min})$ with Vortex mixer, the mixture was heated in the water bath for $2 \mathrm{hrs}$ at $50^{\circ} \mathrm{C}$. The residue was extracted after it was re-mixed and centrifuge at $4600 \mathrm{rpm}$ for $15 \mathrm{mins}$ at $4^{\circ} \mathrm{C}$. The clear supernatant was separated for the analysis of total phenolic contents using the Folin-Ciocalteu colorimetric method (Ainsworth and Gillespie, 2007) and the results were expressed in gallic acid equivalents per gram dry weight (mg GAE/g DW) of the sample. The antioxidant activity was determined using the DPPH-radical scavenging assay (Fukumoto and Mazza, 2000), ORAC (oxygen radical absorbance capacity) assay (Ou et al., 2001) and FRAP assays (Benzie and Strain, 1996) and the results were expressed as Trolox equivalents per $100 \mathrm{~g}$ dry weight (TE/ $100 \mathrm{~g}$ DW) for DPPH assay and Trolox equivalents per gram dry weight (TE/g DW) for ORAC and FRAP assays, respectively.

\subsection{Statistical analysis}

All measurement except sensory evaluation was performed in triplicates. Experimental data were analyzed using computer software (IBM SPSS Statistics 19.0 IBM, Chicago, Illinois, USA). Independent sample t -test or one-way Analysis of Variance (ANOVA) and Duncan's multiple range tests was performed to compare mean value. Average values are considered significantly different when $\mathrm{p}<0.05$.

\section{Results and discussion}

\subsection{Selection of the control crispy waffle formula}

The sensory evaluation of three different crispy waffle formulas is shown in Table 2. The results indicated that no significant differences were found in appearance, color, flavor and taste between formula B and $\mathrm{C}$ while formula $\mathrm{A}$ showed a significant difference with formula $\mathrm{B}$ and $\mathrm{C}$. These might be due to formula A contained yeast. Yeast plays a vital role in dough expansion during fermentation due to $\mathrm{CO}_{2}$ production along with the development of flavor and alcohol synthesis (Ali et al., 2012). In addition, the liking score of texture and overall liking of these three crispy waffle formulas were not significantly different. However, formula $\mathrm{C}$ obtained the highest overall liking score and offered the lowest cost. Therefore, formula $\mathrm{C}$ was selected to be the control formula for the development of a low GI crispy waffle. 
Table 2. Sensory evaluation of three different crispy waffle formulas.

\begin{tabular}{lccc}
\hline \multirow{2}{*}{ Sensory attributes } & \multicolumn{3}{c}{ Formulas } \\
\cline { 2 - 4 } & $\mathrm{A}$ & $\mathrm{B}$ & $\mathrm{C}$ \\
\hline Appearance & $6.67 \pm 1.40^{\mathrm{b}}$ & $7.53 \pm 0.86^{\mathrm{a}}$ & $7.26 \pm 0.68^{\mathrm{a}}$ \\
Color & $6.47 \pm 1.43^{\mathrm{b}}$ & $7.40 \pm 0.93^{\mathrm{a}}$ & $7.57 \pm 0.73^{\mathrm{a}}$ \\
Flavor & $6.77 \pm 1.38^{\mathrm{b}}$ & $7.10 \pm 0.96^{\mathrm{a}}$ & $7.47 \pm 0.86^{\mathrm{a}}$ \\
Taste & $6.63 \pm 1.40^{\mathrm{b}}$ & $6.93 \pm 1.28^{\mathrm{b}}$ & $7.33 \pm 0.80^{\mathrm{a}}$ \\
Texture & $6.83 \pm 1.18^{\mathrm{a}}$ & $7.07 \pm 1.14^{\mathrm{a}}$ & $6.93 \pm 1.41^{\mathrm{a}}$ \\
Overall liking & $6.97 \pm 1.22^{\mathrm{a}}$ & $7.07 \pm 1.01^{\mathrm{a}}$ & $7.50 \pm 1.04^{\mathrm{a}}$ \\
\hline
\end{tabular}

Values are expressed as mean \pm SD. Values with different superscript indicate significant difference at $\mathrm{p}<0.05$ using one way ANOVA followed by Duncan multiple range test.

\subsection{Effect of RS IV substitution on physical and sensory properties of WF crispy waffle replaced with RS IV}

Three different levels $(35,45$ and 55\% (w/w)) of RS IV were used to replace WF in low GI crispy waffle. The physical and sensory properties of the low GI crispy waffle formulas are shown in Table 3 . The water activity was slightly decreased with the increasing of RS IV. The water activity of samples was in the ranges of $0.08-0.11$. In fact, yeast and mold start to grow at water activity in the range between 0.70 and 0.80 (Rawat and Darappa, 2015). The samples are classified as a low moisture food product (LMF) due to the water activity value being below 0.70 . The low water activity of the samples would help to extend its shelf life (Farakos et al., 2013). The color measurement indicated that the increase of RS IV resulted in the increasing of the $L^{*}$ value while $a^{*}$ and $b^{*}$ values were decreased. This meant that the higher amount of RS IV replacement made the samples is lighter. This was could be due to the color of RS IV which is white while the color of WF is yellow (Aramouni and Mahmoud, 2011). In addition, the hardness of low GI crispy waffles was significantly different from the variation of RS IV content but no significant difference of fracturability was found. This could be due to the additional fibers dilute gluten and distort the gluten-starch network formed (Pham et al., 2007). These results were similar with Lestari et al. (2017) who found that the higher addition of arrowroot flour resulted in the more easily crumbled texture since this product contains the highest dietary fiber and RS.

In case of sensory evaluation, no significant differences were found in appearance, color, flavor and taste between low GI crispy waffles with 45 and $55 \%$ (w/ w) of RS IV while low GI crispy waffle with $35 \%(\mathrm{w} / \mathrm{w})$ RS IV showed the significant difference with $55 \%(\mathrm{w} / \mathrm{w})$ RS IV. The texture and overall liking scores were not significantly different from the variation of RS IV content. Therefore, the low GI crispy waffle with $55 \%$ (w/w) RS IV was selected to be the formula used to produce low GI waffle. This was due to the high amount of RS IV could provide a high content of fiber in the product and result in health benefit. As reported by Stewart et al. (2018) found that the replacement WF with RS IV in scone can increase fiber in the product and improve postprandial glucose and insulin sensitivity.

\subsection{Feasibility study of fortification of sacred lotus stamen powder (SLSP) in low GI crispy waffle}

Table 4 presents the physical and sensory properties of low GI crispy waffle fortified with SLSP at 30, 35 and $40 \%(\mathrm{w} / \mathrm{w})$ for WF. The results showed that with the increasing amount of SLP, the water activity increases.

Table 3. Physical and sensory properties of low GI crispy waffle replaced with three different levels of RS IV.

\begin{tabular}{lccc}
\hline \multirow{2}{*}{ Properties } & \multicolumn{3}{c}{ Type IV resistant starch (RS IV) (\%) } \\
\cline { 2 - 4 } & 35 & 45 & 55 \\
\hline Water activity & $0.11 \pm 0.01^{\mathrm{a}}$ & $0.09 \pm 0.00^{\mathrm{ab}}$ & $0.08 \pm 0.00^{\mathrm{b}}$ \\
Color - $L^{*}$ & $42.06 \pm 0.10^{\mathrm{c}}$ & $42.55 \pm 0.03^{\mathrm{b}}$ & $43.34 \pm 0.03^{\mathrm{a}}$ \\
- $^{*}$ & $5.64 \pm 0.02^{\mathrm{a}}$ & $5.56 \pm 0.01^{\mathrm{b}}$ & $5.46 \pm 0.02^{\mathrm{c}}$ \\
- $^{*}$ & $14.77 \pm 0.03^{\mathrm{a}}$ & $14.73 \pm 0.06^{\mathrm{a}}$ & $14.61 \pm 0.03^{\mathrm{b}}$ \\
Hardness (g-force) & $1330.16 \pm 149.49^{\mathrm{a}}$ & $1101.54 \pm 140.02^{\mathrm{b}}$ & $827.57 \pm 100.66^{\mathrm{c}}$ \\
Fracturability (mm-distance) & $0.90 \pm 0.44^{\mathrm{a}}$ & $0.85 \pm 0.38^{\mathrm{a}}$ & $0.69 \pm 0.49^{\mathrm{a}}$ \\
\hline Sensory evaluation & & & \\
\hline Appearance & $7.30 \pm 0.95^{\mathrm{b}}$ & $7.37 \pm 0.96^{\mathrm{a}}$ & $7.46 \pm 1.16^{\mathrm{a}}$ \\
Color & $7.23 \pm 0.94^{\mathrm{b}}$ & $7.53 \pm 0.97^{\mathrm{a}}$ & $7.67 \pm 1.15^{\mathrm{a}}$ \\
Flavor & $7.00 \pm 0.82^{\mathrm{b}}$ & $7.13 \pm 1.04^{\mathrm{ab}}$ & $7.90 \pm 0.84^{\mathrm{a}}$ \\
Taste & $7.37 \pm 0.81^{\mathrm{b}}$ & $7.40 \pm 0.93^{\mathrm{ab}}$ & $7.83 \pm 0.95^{\mathrm{a}}$ \\
Texture & $7.23 \pm 0.86^{\mathrm{a}}$ & $7.43 \pm 0.94^{\mathrm{a}}$ & $7.73 \pm 1.23^{\mathrm{a}}$ \\
Overall liking & $7.33 \pm 0.88^{\mathrm{a}}$ & $7.37 \pm 0.85^{\mathrm{a}}$ & $7.90 \pm 1.06^{\mathrm{a}}$ \\
\hline
\end{tabular}

Values are expressed as mean \pm SD. Values with different superscript indicate significant difference at $\mathrm{p}<0.05$ using one way ANOVA followed by Duncan multiple range test. 
Table 4. Physical and sensory properties of low GI crispy waffle fortified with three different levels of SLSP.

\begin{tabular}{lccc}
\hline \multirow{2}{*}{ Properties } & \multicolumn{3}{c}{ Sacred lotus stamen powder (SLSP) $\%$ ) } \\
\cline { 2 - 4 } & 30 & 35 & 40 \\
\hline Water activity & $0.14 \pm 0.01^{\mathrm{c}}$ & $0.16 \pm 0.00^{\mathrm{b}}$ & $0.20 \pm 0.00^{\mathrm{a}}$ \\
Color - $L^{*}$ & $35.63 \pm 0.04^{\mathrm{a}}$ & $35.71 \pm 0.05^{\mathrm{a}}$ & $35.52 \pm 0.05^{\mathrm{b}}$ \\
- $^{*}$ & $4.97 \pm 0.01^{\mathrm{c}}$ & $5.01 \pm 0.02^{\mathrm{b}}$ & $5.36 \pm 0.01^{\mathrm{a}}$ \\
- $^{*}$ & $17.40 \pm 0.12^{\mathrm{b}}$ & $17.29 \pm 0.04^{\mathrm{b}}$ & $17.75 \pm 0.07^{\mathrm{a}}$ \\
Hardness (g-force) & $1377.51 \pm 116.95^{\mathrm{b}}$ & $1645.43 \pm 126.49^{\mathrm{a}}$ & $1601.70 \pm 122.22^{\mathrm{a}}$ \\
Fracturability (mm-distance) & $0.50 \pm 0.24^{\mathrm{a}}$ & $0.66 \pm 0.24^{\mathrm{a}}$ & $0.51 \pm 0.13^{\mathrm{a}}$ \\
\hline Sensory evaluation & & & \\
\hline Appearance & $6.47 \pm 1.04^{\mathrm{a}}$ & $6.67 \pm 0.92^{\mathrm{a}}$ & $6.90 \pm 1.12^{\mathrm{a}}$ \\
Color & $6.37 \pm 1.16^{\mathrm{a}}$ & $6.40 \pm 1.10^{\mathrm{a}}$ & $6.50 \pm 1.13^{\mathrm{a}}$ \\
Flavor & $6.13 \pm 1.46^{\mathrm{b}}$ & $6.40 \pm 116^{\mathrm{ab}}$ & $7.00 \pm 1.05^{\mathrm{a}}$ \\
Taste & $6.03 \pm 1.43^{\mathrm{b}}$ & $6.60 \pm 0.93^{\mathrm{ab}}$ & $6.97 \pm 1.07^{\mathrm{a}}$ \\
Texture & $6.33 \pm 1.27^{\mathrm{a}}$ & $6.57 \pm 1.10^{\mathrm{a}}$ & $6.87 \pm 1.17^{\mathrm{a}}$ \\
Overall liking & $6.20 \pm 1.49^{\mathrm{c}}$ & $6.53 \pm 1.07^{\mathrm{b}}$ & $7.03 \pm 1.33^{\mathrm{a}}$ \\
\hline
\end{tabular}

Values are expressed as mean \pm SD. Values with different superscript indicate significant difference at $p<0.05$ using one way ANOVA followed by Duncan multiple range test.

However, the water activity of all formulas was lower than 0.65 which indicated the product was stable for microbial growth (Farakos et al., 2013). The low GI waffle with $40 \%(\mathrm{w} / \mathrm{w})$ SLSP had the greatest redness $\left(a^{*}\right)$ and yellowness $\left(b^{*}\right)$ than low GI crispy waffle with 30 and $35 \%(\mathrm{w} / \mathrm{w})$ SLSP. This was due to the color of SLSP. The hardness value of samples increased with increasing of SLSP content. This might be due to the addition of SLSP interfered the development of gluten complex. The results are in agreement with Lestari et al. (2017) who reported that the higher addition of kidney beans resulted in the harder cookie bars. However, no significant difference was found on fracturability.

The sensory evaluation results presented that there were no significant differences in the score of appearance, color and texture between three formulas. The flavor and taste scores of low GI crispy waffle fortified with $30 \%(\mathrm{w} / \mathrm{w})$ SLSP were significantly lower than with $40 \%(\mathrm{w} / \mathrm{w})$ SLSP but no significant difference was found with $35 \%(\mathrm{w} / \mathrm{w})$ SLSP. This might be due to the unique taste and flavor of SLSP (Wongkhlang, 2015). Finally, low GI crispy waffle fortified with $40 \%$ $(\mathrm{w} / \mathrm{w})$ SLSP obtained the significantly highest overall liking score. Thus, low GI crispy waffle fortified with $40 \% \quad(\mathrm{w} / \mathrm{w})$ SLSP was selected and subsequently determined nutritional values, GI, TPCs and antioxidant activities.

\subsection{Nutritional values, Glycemic index, total phenolic contents and antioxidant activities}

The nutritional values, GI, TPCs and antioxidant activities of the control crispy waffle (without RS IV and SLSP) and low GI crispy waffle fortified with $40 \%$ (w/ w) SLSP are shown in Table 5. The total carbohydrate and protein of the low GI crispy waffle with SLSP were decreased from 68.43 to $64.52 \mathrm{~g}$ and 10.24 to $6.63 \mathrm{~g}$, respectively. This was due to the replacement of WF with RS IV and SLSP which contains lower carbohydrate and protein content than WF (Eliasson and Cauvain; 2012; Cui et al., 2013; Parinya et al., 2018). However, energy, total fat, dietary fiber and ash of low GI crispy waffle with SLSP were increased. The increasing of fiber in the developed product as expected due to RS IV and SLSP is a source of fiber (Fan, 2017; Erickson et al., 2018). It observed that fiber content of low GI crispy waffle with SLSP was $28 \%$ Thai RDI. Therefore, this product can be claimed as high fiber as according to Thai FDA regulation on nutrition labelling. The glycemic index of low GI crispy waffle with SLSP was determined using glycemic index (eGI) analysis. GI of the developed crispy waffle (28.8) was lower than the control formula (31.4). This could be due to fat and dietary fiber inhibit sugar absorption (Moghaddam et al., 2006; Odenigbo et al., 2012). The result was similar to Lestari et al. (2017) who found that GI of cookie bar was decreased after formulation with foxtail millet, arrowroot flour, and kidney beans. Finally, the developed crispy waffle was classified as low GI product (GI£55).

There are numerous published methods measuring total antioxidant capacity in vitro, which can be classified into two types: assays based on hydrogen atom transfer (HAT) and assays based on electron transfer (ET). HAT-based assays, like the ORAC assay, apply a competitive reaction scheme, in which antioxidant and substrate compete for thermally generated peroxyl radicals. ET-based assays measure the capacity of an 
Table 5. Nutritional values, GI, TPCs and antioxidant activities of the control and developed crispy waffle formulas.

\begin{tabular}{|c|c|c|}
\hline \multirow{2}{*}{ Properties } & \multicolumn{2}{|r|}{ Formulas } \\
\hline & Control crispy waffle & Low GI crispy waffle with SLSP \\
\hline Energy (kcal/100 g) & $478.42 \pm 1.14$ & $491.22 \pm 4.06$ \\
\hline Total carbohydrate (g/100 g) & $68.43 \pm 0.23^{*}$ & $64.52 \pm 0.61$ \\
\hline Protein (g/100 g) & $10.24 \pm 0.06^{*}$ & $6.63 \pm 0.06$ \\
\hline Total fat (g/100 g) & $18.20 \pm 0.21$ & $22.96 \pm 0.75^{*}$ \\
\hline Dietary fiber $(\mathrm{g} / 100 \mathrm{~g})$ & $3.23 \pm 0.07$ & $28.04 \pm 0.18^{*}$ \\
\hline Ash (g/100 g) & $0.67 \pm 0.02$ & $1.14 \pm 0.01^{*}$ \\
\hline Glycemic index (GI) & 31.4 & 28.8 \\
\hline TPCs (mg GAE/g DW) & $1.12 \pm 0.07$ & $2.30 \pm 0.05^{*}$ \\
\hline \multicolumn{3}{|l|}{ Antioxidant activities } \\
\hline DPPH radical scavenging assay ( $\mu$ mol TE/100 g DW) & $0.16 \pm 0.01$ & $0.76 \pm 0.07^{*}$ \\
\hline FRAP assay ( $\mu$ mol TE/g DW) & $1.83 \pm 0.13$ & $7.33 \pm 0.38^{*}$ \\
\hline ORAC assay ( $\mu \mathrm{mol}$ TE/g DW) & $13.16 \pm 0.92$ & $56.45 \pm 4.22^{*}$ \\
\hline
\end{tabular}

Values are expressed as mean \pm SD. $*$ means significant difference at $\mathrm{p}<0.05$ using student's t-test.

antioxidant to reduce an oxidant, which changes color when reduced. The degree of color change is correlated with the sample's antioxidant concentration. ET-based assays such as TPCs by Folin-Ciocalteu reagent, DPPH and FRAP assay. No single method is sufficient; more than one type of antioxidant capacity measurement needs to be performed to take into account the various modes of action of antioxidants (Prior and Cao, 1999; Huang et al., 2005). In this study, free radical scavenging capacities of the extracts using DPPH, and ORAC assays, and their ferric reducing capacities using the FRAP assay were done. The results showed that TPCs and antioxidant activities (by DPPH, FRAP and ORAC assays) of low GI crispy waffle with SLSP was $2.30 \mathrm{mg}$ GAE/g DW, $0.76 \mu \mathrm{mol}$ TE/100 g DW, $7.33 \mu \mathrm{mol} \mathrm{TE} / \mathrm{g}$ DW and $56.45 \mu \mathrm{mol}$ TE/g DW, respectively. Moreover, TPCs and antioxidant activities of the developed crispy waffle was higher than the control formula. As Lockyer and Nugent (2017) reported that the TPCs and antioxidant activities were not found in RS IV. Therefore, it might confirm that the addition of SLSP in the product resulted in a healthier product with functional properties. In addition, these data suggested that TPCs was positively correlated with antioxidant activities which were found in many plants (Ghanbari et al., 2015). According to the cost calculation, the cost of developed crispy waffle was higher than the control formula which was due to the higher price of RS IV and RLSP than WF. However, when considering their health benefits, this developed crispy waffle could be an alternative product for people with diabetes or normal people.

\section{Conclusion}

This study revealed that the product qualities of crispy waffle were slightly affected by the chemical composition of ingredients. The 55\% (w/w) RS IV and
$18 \%(\mathrm{w} / \mathrm{w})$ SLSP in substitution of WF significantly enhances fiber, TPCs and antioxidant activities of the developed crispy waffle. The developed crispy waffle had a lower GI than the control crispy waffle. Therefore, RS IV and SLSP could be the interesting functional ingredients used to improve nutritional values and antioxidant activities of other food formulation.

\section{Conflict of interest}

The authors have no potential conflict of interest.

\section{Acknowledgements}

This work was supported by Institute nutrition Mahidol University and funded by Capacity Building Program for New Researcher 2019 from National Research Council of Thailand (NRCT).

\section{References}

Ainsworth, E.A. and Gillespie, K.M. (2007). Estimation of total phenolic content and other oxidation substrates in plant tissues using Folin-Ciocalteu reagent. Nature Protocols, 2, 875-877. https:// doi.org/10.1038/nprot.2007.102

Ali A., Shehzad, A., Khan, M.R., Shabbir, M.A., Amjid, M.R. (2012). Yeast, its types and role in fermentation during bread making process-A Review, Pakistan Journal of Food Sciences, 22(3), 171-179.

AOAC. (2016). Official methods of analysis of AOAC International. $20^{\text {th }}$ ed. Gaithersburg: MD, USA.

Aramouni, F. and Mahmoud, A. (2011). Physicochemical and sensory characteristics of nobake wheat-soy snack bars. Journal of Science of Food and Agriculture, 91(1), 44-51. https:// doi.org/10.1002/jsfa.4134 
Baixauli, R., Salvador, A., Martínez-Cervera, S. and Fiszman, S.M. (2008). Distinctive sensory features introduced by resistant starch in baked products. LWT-Food Science and Technology, 41(10), 19271933. https://doi.org/10.1016/j.lwt.2008.01.012

Benzie, I. and Strain, J. (1996). The ferric reducing ability of plasma (FRAP) as a measure of antioxidant power: The FRAP assay. Analytical Biochemistry, 239(1), 70-76. https://doi.org/10.1006/ abio.1996.0292

Birt, D.F., Boylston, T., Hendrich, S., Jane, J.-L., Hollis, J., Li, L., McClelland, J., Moore, S., Phillips, G.J., Rowling, M. Schalinske, K., Scott, M.P. and Whitley, M. (2013). Resistant starch: Promise for improving human health. Advances in Nutrition, 4 (6), 587-601. https://doi.org/10.3945/an.113.004325

Brand-Miller, J., Dickinson, S., Barclay, A. and Celermajer, D. (2007). The glycemic index and cardiovascular disease risk. Atherosclerosis Reports, 9, 479-485. https://doi.org/10.1007/s11883-007-0064 $-\mathrm{x}$

Charalampopoulos, D., Wang, R., Pandiella, S.S. and Webb, C. (2002). Application of cereals and cereal components in functional foods: A review. International Journal of Food Microbiology, 79(12), 131-141. https://doi.org/10.1016/S0168-1605(02) 00187-3

Choi, Y.J., Kim, H.C., Kim, H.M., Park, S.W., Kim, J. and Kim, D.J. (2009). Prevalence and management of diabetes in Korean adults. Diabetes care, 32(11), 2016-2020. https://doi.org/10.2337/dc08-2228

Cui, S.W., Wu, Y. and Ding, H. (2013). The range of dietary fiber ingredients and a comparison of their technical functionality. In Delcaour, J.A and Poutanen, K. (Eds.) Fiber-Rich and Wholegrain Foods., p. 96-119. USA: Woodhead Publishing Limited. doi.org/10.1533/9780857095787.1.96

Eliasson, A.C. and Cauvain, S.P. (2012). 6 - Wheat starch structure and bread quality. In Cauvain, S.P. (Ed.). Breadmaking. $2^{\text {nd }}$ ed., p. $123-148$. USA: Woodhead Publishing Limited. https:// doi.org/10.1533/9780857095695.1.123

Erickson, J.M., Carlson, J.L., Stewart, M.L. and Slavin, J.L. (2018). Fermentability of novel type-4 resistant starches in in vitro system. Foods, 7(2), 18. https:// doi.org/10.3390/foods 7020018

Fan, Z. (2017). Review structures, properties, and applications of lotus starches. Food Hydrocolloids, 63, 332-348. https://doi.org/10.1016/ j.foodhyd.2016.08.034

Farakos, S.S., Frank, J., and Schaffner, D. (2013).
Modeling the influence of temperature, water activity and water mobility on the persistence of salmonella in low-moisture foods. International Journal of Food Microbiology, 166(2), 280-293. https://doi.org/10.1016/j.jifoodmicro.2013.07.007

Fukumoto, L.R. and Mazza, G. (2000). Assessing antioxidant and proxidant activities of phenolic compounds. Journal of Agricultural and Food Chemistry, 48(8), 3597-3604. https:// doi.org/10.1021/jf000220w

Ghanbari, R., Zarei, M., Ebrahimpour, A., Abdul-Hamid, A., Ismail, A. and Saari, N. (2015). Angiotensin-I converting enzyme (ACE) inhibitory and antioxidant activities of sea cucumber (Actinopyga lecanora) hydrolysates. International journal of Molecular Sciences, 16(12), 28870-28885. https:// doi.org/10.3390/ijms161226140

Granfeldt, Y., Liljeberg, H., Drews, A., Newman, R. and Bjorck, I. (1994). Glucose and insulin responses to barley products influence of food structure and amylose-amylopectin ratio. American Journal Clinical Nutrition, 59(5), 1075-1082. https:// doi.org/10.1093/ajen/59.5.1075

Homayouni, A., Amini, A., Keshtiban, A.K., Mortazavian, A.M., Esazadeh, K. and Pourmoradian, S. (2014). Resistant starch in food industry: A changing outlook for consumer and producer. Starch Stärke, 66(1-2), 102-114. https://doi.org/10.1002/ star.201300110

Huang, D., Ou, B. and Prior, R.L. (2005). The chemistry behind antioxidant capacity assays. Journal of Agricultural and Food Chemistry, 53, 1841-1856. https://doi.org/10.1021/jf030723c

Hung, H.C., Joshipura, K.J., Jiang, R., Hu, F.B., Hunter, D., Smith-Warner, S.A., Colditz, G.A., Rosner, B., Spiegelman, D. and Willett, W.C. (2004). Fruit and vegetable intake and risk of major chronic disease. Journal of the National Cancer Institute, 96(21), 1577-1584. https://doi.org/10.1093/jnci/djh296

Jambrec, D., Pestorić, M., Sakač, M., Nedeljković, N., Hadnađev, M., Filipčev, B. and Šimurina, O. (2013). Sensory and instrumental properties of novel glutenfree products. Journal Process of Agriculture, 17(2), 86-88.

Jung, H.A., Kim, J.E., Chung, H.Y. and Choi, J.S. (2003). Antioxidant principles of Nelumbo nucifera stamens. Archives of Pharmacal Research, 26(4), 279-285. https://doi.org/10.1007/BF02976956

Lestari, LA., Huriyati, E. and Marsono, Y. (2017). The development of low glycemic index cookie bars from foxtail millet (Setaria italica), arrowroot (Maranta arundinacea) flour, and kidney beans 
(Phaseolus vulgaris). Journal Food Science Technology, 54(6), 1406-1413. https:// doi.org/10.1007/s13197-017-2552-5

Lockyer, S. and Nugent, A.P. (2017). Health effects of resistant starch. Food and Nutrition Bulletin, 42(1), 10-41. https://doi.org/10.1111/nbu.12244

Meilgaard, M., Civille G.V. and Carr, B.T. (1999). Sensory evaluation techniques. $3^{\text {rd }}$ ed. Boca Raton: CRC press. https://doi.org/10.1201/9781439832271

Moghaddam, E., Vogt, J.A. and Wolever, T.M.S. (2006). The effects of fat and protein on glycemic responses in nondiabetic humans vary with waist circumference, fasting plasma insulin, and dietary fiber intake. The Journal of Nutrition, 136(10), 25062511. https://doi.org/10.1093/jn/136.10.2506

Nutrition Subcommittee of the Diabetes Care Advisory Committee of Diabetes UK. (2003). The implementation of nutritional advice for people with diabetes. Diabetic Medicine Journal, 20(10), 786807. https://doi.org/10.1046/j.14645491.2003.01104.x

Odenigbo, A., Rahimi, J., Ngadi, M., Amer, S. and Mustafa, A. (2012). Starch digestibility and predicted glycemic index of fried sweet potato cultivars. Functional Foods in Health and Disease, 2 (7), 280-289. https://doi.org/10.31989/ffhd.v2i7.83

Office of Nutrition and Food Labeling, Center for Food Safety and Applied Nutrition, Food and Drug Administration, US Department of Health and Human Services. (2016). Science Review of Isolated and Synthetic Non-Digestible Carbohydrates. Retrieved from FDA website: https://www.fda.gov/ media/101853/download

Ou, B., Hampsch-Woodill, M. and Prior, R. (2001). Development and validation of an improved oxygen radical absorbance capacity assay using fluorescein as the fluorescent probe. Journal of Agricultural and Food Chemistry, 49(10), 4619-4626. https:// doi.org/10.1021/jf010586o

Parinya, T., Phomphang, U., Deesnam, N. and Pongjanta, J. (2018). Physicochemical properties of low glycemic index-high fiber rice flour from storage rice grain and application on chocolate chip cookies as substitute for wheat flour. Current Applied Science and Technology, 18(1), 1-11.

Perlstein, R.W.J., Hines, C. and Milsavljevic, M. (1997). Dieticians Association of Australia review paper: Glycaemic index in diabetes management. Australian Journal of Nutrition Dietetics, 54, 57-63.

Pham, V.H., Tomoko, M. and Naofumi, M. (2007). Dough and bread qualities of flours with whole waxy wheat flour substitution. Food Research
International, $\quad 40(2), \quad 273-279 . \quad$ https:// doi.org/10.1016/j.foodres.2006.10.007

Prior, R.L. and Cao, G. (1999). In vivo total antioxidant capacity: comparison of different analytical methods. Free Radical and Biological Medicine, 27(11-12), 1173-1181. https://doi.org/10.1016/S0891-5849(99) 00203-8

Rawat, N. and Darappa, I. (2015). Effect of ingredients on rheological, nutritional and quality characteristics of fiber and protein enriched baked energy bars. Journal of Food Science and Technology, 52(5), 3006-3013. https://doi.org/10.1007/s13197-014-1367 $-\mathrm{X}$

Soerjomataram, I., Oomen, D., Lemmens, V., Oenema, A., Benetou, V., Trichopoulou, A., Coebergh, J.W., Barendregt, J. and De Vries, E. (2010). Increased consumption of fruit and vegetables and future cancer incidence in selected european countries. European Journal of Cancer, 46(14), P2563-P2580. https://doi.org/10.1016/j.ejca.2010.07.026

Stewart, M.L., Wilcox, M.L., Marjorie, B., Buggia, M.A. and Maki K.C. (2018). Type-4 resistant starch in substitution for available carbohydrate reduces postprandial glycemic response and hunger in acute, randomized, double-blind, controlled study. Nutrients, 10(2), 129. https://doi.org/10.3390/ nu10020129

Wang, S., Melnyk, J.P., Tsao, R. and Marcone, M.F. (2011). How natural dietary antioxidants in fruits, vegetables and legumes promote vascular health. Food Research International, 44(1), 14-22. https:// doi.org/10.1016/j.foodres.2010.09.028

Wongkhlang, S. (2015). Antioxidant activity of sacred lotus (Nelumbo nucifera) for development of cereal bar product. Thailand: Rajamangala University of Technology Thanyaburi, MSc. Thesis. 